\title{
Rare Non-Neoplastic Disorder
}

National Cancer Institute

\section{Source}

National Cancer Institute. Rare Non-Neoplastic Disorder. NCI Thesaurus. Code C53543.

A non-neoplastic disorder that is rare. 